Presented at the 1978 IEEE Nuclear

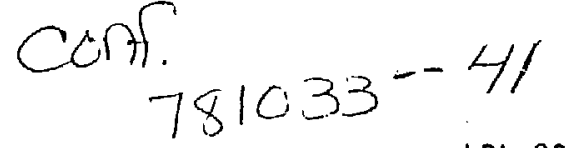

Science Symposium, Washington D. C.,

October 18-20, 1978

\title{
RADIATION DAMAGE RESISTANCE OF REVERSE \\ ELECTRODE GE COAXIAL DETECTORS
}

Richard H. Pehl, Norman W. Madden, Jack H. Elliott, Thomas W. Raudorf, Rex C. Tramme11 and Lawrence S. Darken, Jr.

October 1978

Prepared for the U. S. Department of Energy under Contract $W-7405-E N G-48$

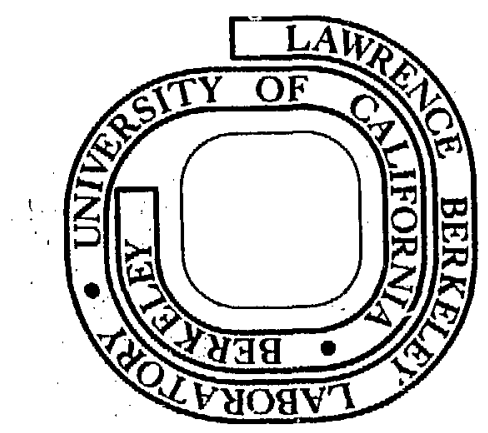


Richard H. Pehl*, Norman W. Madden*, Jack H. Elliott**

Thomas W. Raudorf ${ }^{+}$, Rex C. Trame $11^{+}$and Lawrence S. Darken, Jr. ${ }^{+}$

\section{Summary}

Two high-purity germaniuni coaxial detectors, having opposite electrode configurations from one another, but fabricated from the same gerinanium crystal, were irradiated simultaneously with fast neutrons from an unmoderated ${ }^{252} \mathrm{Cf}$ source. Both detectors were $42 \mathrm{~mm}$ diam. The detector having the conventional electrode configuration was about 28 times more sensitive to radiation damage than was the detector having the $\mathrm{p}^{+}$contact on the coaxial periphery.

These results prove that germanium coaxial detectors having the conventional electrode configuration should not be used in any situation subject to significant radiation damage. This conclusion was anticipated because the defects produced by neutron and proton irradiation of germanium act predominantly as hole traps.

\section{Introduction}

It has been known for a number of years that fast neutron irradiation of germanium detectors produces predominately defects which act as hole traps. ${ }^{1}$ This fact leads to consideration of the possibility of minimizing hole trapping in charge collection by the use of a high-purity germanium coaxial detector configured with the $\mathrm{p}^{+}$contact on the periphery of the coaxial structure. ${ }^{2}$

As depicted in Fig. 1, most of the holes produced by gamma-ray interactions will then make only a short traversal from the outer portions of the detector (where most of the interactions occur because of geometry) to the contact. To establish a high electric field at the periphery $n$-type germanium should be used.

*Lawrence Berkeley Laboratory, University of California, Berkeley, California 94720, U.S.A., **l.awrence Livermore Laboratory, University of California, Livermore, California 94550 , U.S.A.

+E.G.\&G. ORTEC, 100 Midland Road, Oak Ridge, Tennessee 37830, U.S.A.

${ }^{++}$This work was supported by the Nuclear Science Division of the Department of Energy under Contract No. W-7405-ENG-48. 
In the conventional high-purity and lithium-drifted germanium coaxial detectors the hole collection process dominates the signal, whereas when the electrode configuration is reverseu as discussed here the electron collection process dominates the signal. Thus a coaxial detector having a reversed electrode configuration should be less sensitive to radiation damage.

In the course of a continuing study of the proton damage of germanium detectors data were obtained that implied an improvement in radiation damage resistance of about 60 times when comparing very large coaxial detectors having the opposite electrode configuration. ${ }^{3,4}$ However, this factor was obtained via a series of indirect comparisons, and was possibly subject to some error. The purnose of the work reported here is to directly test this hypothesis and to determine the magnitude of the improvement.

\section{Experimental}

Two high-purity germanium coaxial detectors that were fabricated from the same germanium crystal grown at EG\&G ORTEC ${ }^{5}$ were irradiated with fast neutrons from an unmoderated ${ }^{252} \mathrm{Cf}$ source at the Lawrence Livermore Laboratory $\mathrm{H} i \mathrm{iza}$ ards Control Standards and Calibrations Laboratory. ${ }^{6}$ In light of the range of radiation damage sensitivities among detectors made from different crystals ${ }^{7}$ it is important that comparisons be made from detectors fabricated from the same crystal if possible. The fortuitous variation of the net electrically active impurity concentration in the crystal illustrated in Fig. 2 allowed the fabrication of coaxial detectors having the opposite electrode configurations from nearly adjacent pieces of material. Only a $4 \mathrm{~mm}$ thick material evaluation slice was taken between the germanium pieces from which the detectors were fabricated. As expected because of the various effective impurity distribution functions, the material for the detector having the conventional electrode configuration came nearer the head of the crystal. Although the two detectors were mounted in separate cryostats cooled with $\mathrm{LN}_{2}$ both detectors were maintained at essentially the same temperature. 
Various detector parameters are listed in Table 1.

Table 1

Detector

\begin{tabular}{ll} 
Conventional & Reverse \\
Electrode & Electrode \\
Configuration & Configuration \\
\hline
\end{tabular}

Diameter

Center Hole Diameter

Length

Relative Efficiency

at $1.332 \mathrm{keV}$

Depletion Bias

Maximum Operating Bias

Operating Bias During

Experiment
$42 \mathrm{~mm}$

$8 \mathrm{~mm}$

$30 \mathrm{~mm}$

$7 \%$

$850 \mathrm{~V}$

$1800 \mathrm{~V}$

$1600 \mathrm{~V}$
$42 \mathrm{~mm}$

$8 \mathrm{~mm}$

$30 \mathrm{~mm}$

$7 \%$

$2600 \mathrm{~V}$

$3000 \mathrm{~V}$

$2800 \mathrm{~V}$

Resolutions for the $1332 \mathrm{keV}{ }^{60} \mathrm{Co}$ line, measured in the experimental configuration at the Standards and Calibrations Laboratory prior to the start of the irradiation, were 1.8 and $2.0 \mathrm{keV}$ (LBL amplifier, $4 \mu \mathrm{s}$ peaking time) for the reverse and conventional electrode configuration detectors respectively. The small difference arose because of additional pick-up that could not be eliminated at the time. The pulser resolutions were 1.0 and $1.3 \mathrm{keV}$, respectively. The ${ }^{252}$ Cf source gave off $1.26 \times 10^{9}$ neutrons per sec, thus with the detectors located $25 \mathrm{~cm}$ from the source they received a flux of $9.63 \times 10^{6}$ $\mathrm{n} / \mathrm{cm}^{2}$ per minute. The experimental plan was to irradiate the detectors simultaneously with a given neutron fluence, then measure the resolution of the gamma-ray lines from a ${ }^{60} \mathrm{Co}$ source. Then a further irradiation would be made, and the resolution measured again. This procedure was followed until the magnitude of the difference in sensitivity to radiation damage between the two detectors was clearly determined. 


\section{Results And Discussion}

Figures 3 and 4 convey the crux of the measurements. Little more needs to be said other than emphasizing the magnitude of the huge difference in sensitivity to radiation damage. The coaxial detector having the reverse electrode configuration is about 28 times less sensitive to the fast neutrons from the ${ }^{252} \mathrm{Cf}$ source, i.e. this detector will withstand 28 times greater neutron fluence before suffering equivalent energy resolution degradation. This difference would he even greater if the coaxial detectors were fabricated from larger diameter crystals. Thus, these results are in essentially perfect agreement with the predicted decrease in sensitivity to radiation damage of 60 times when comparing very 1 arge, approx. $60 \mathrm{~mm}$ diam., coaxial detectors having the opposite electrode configurations. ${ }^{4}$

The evidence is now overwhelming that germanium coaxial detectors having the conventionai electrode configuration should not be used in any situation where they are subject to significant radiation damage. The growing availability of germanium coaxial detectors having the reverse electrode configuration should make the use of 1 arge germanium detectors viable for many additional applications that were previousiy marginal or impractical because of radiation damage problems.

\section{Acknowledgment}

Eugene Haller, Fred Goulding and Larry Varnell provided useful discussions.

\section{$\underline{\text { References }}$}

1. H. W. Kraner, C. Chasman and K. W. Jones, Nucl. Inst. and Meth. 62, 173, 1968.

2. R. H. Peh1, "Germanium Gamma-Ray Detectors", Physics Today, 30, 50, 1977.

3. R. H. Peh1, L. S. Varnel1 and R. H. Parker, "Radiation Damage of Germanium Detectors", Bu11. Am. Phys. Soc. 23, 71, 1978.

4. R. H. Peh1, "Gamma-Ray Spectroscopy in Astrophysics", NASA Technical Memorandum 79619, 473, August 1978. 
5. T. W. Raudorf, R. C. Tramell and L. S. Darken, Jr., to be published in the IEEE Trans. Nuc1. Sci. NS-26, No. 1, 1979.

6. J. H. Elliott, "Tour of the Standards and Calibrations Laboratory", UCRL-52537, 1978.

7. H. W. Kraner, R. H. Pehl and E. E. Haller, IEEE Trans. Nucl. Sci. NS-22, 149, 1975. 


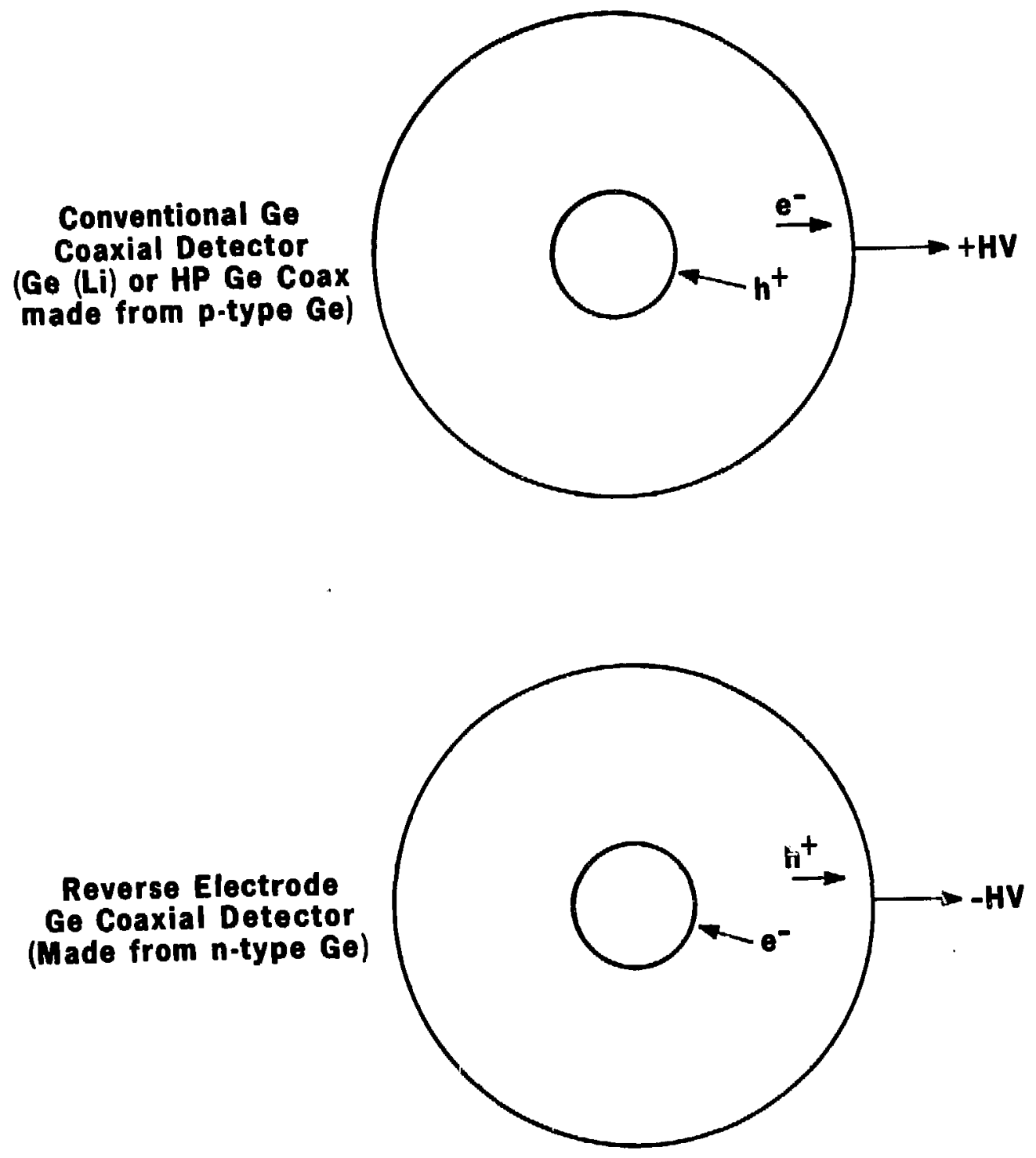

Fig. 1. Charge collection directions for holes and electrons in the conventional Ge coaxial detector and in the reverse

XBL 787-9757 electrode fie coaxial detector. 


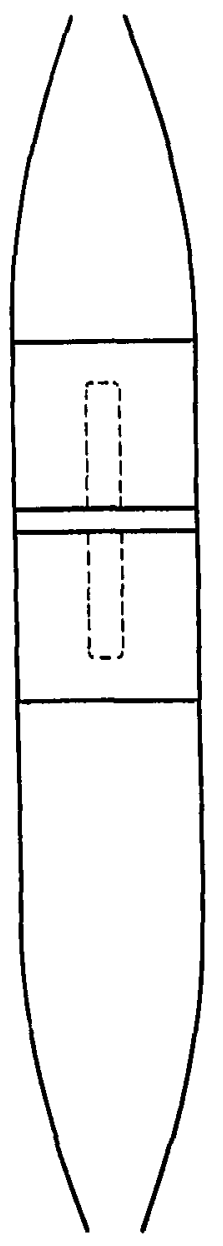

\section{Ge piece for Conventional Electrode Configuration Detector}

\section{Ge piece for Reverse Electrode Configuration Detector}

XBL $7810-12010$

Fiq. 2. Schematic illustration of the qermanium crystal showing the location of the pieces from which the two coaxial tetectors were fabricated. 


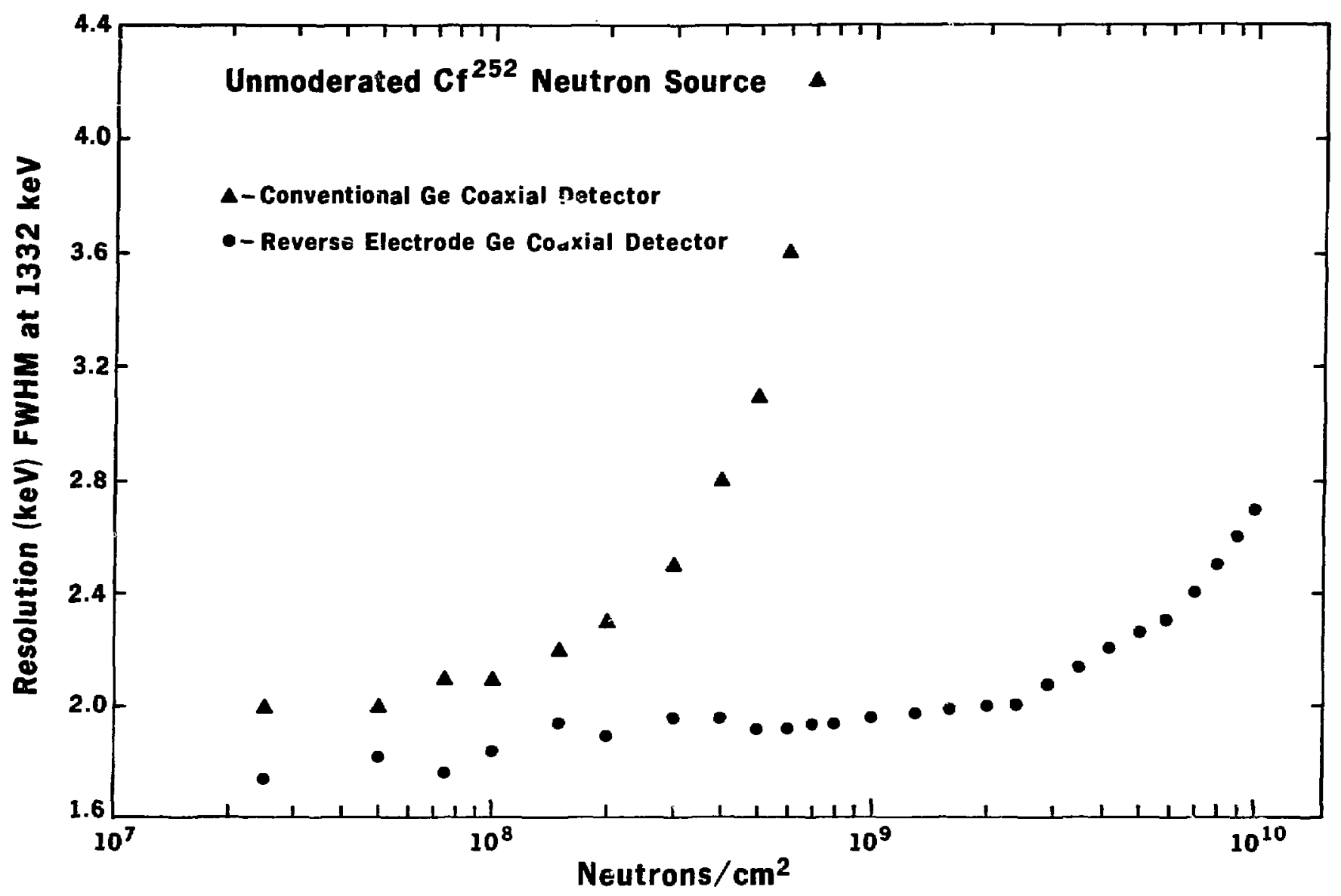

Fia. 3. Effect of jeutron fluence on the enerniv resnlution (Fill!) of the XBL 7810-11755 ! 332 kel ${ }^{67} \mathrm{C}_{\mathrm{C}}$ ! ine for both the convantional and reverse electrode conficurtior. fe coaxial detectors. Electronic noise has not been subtractej. 


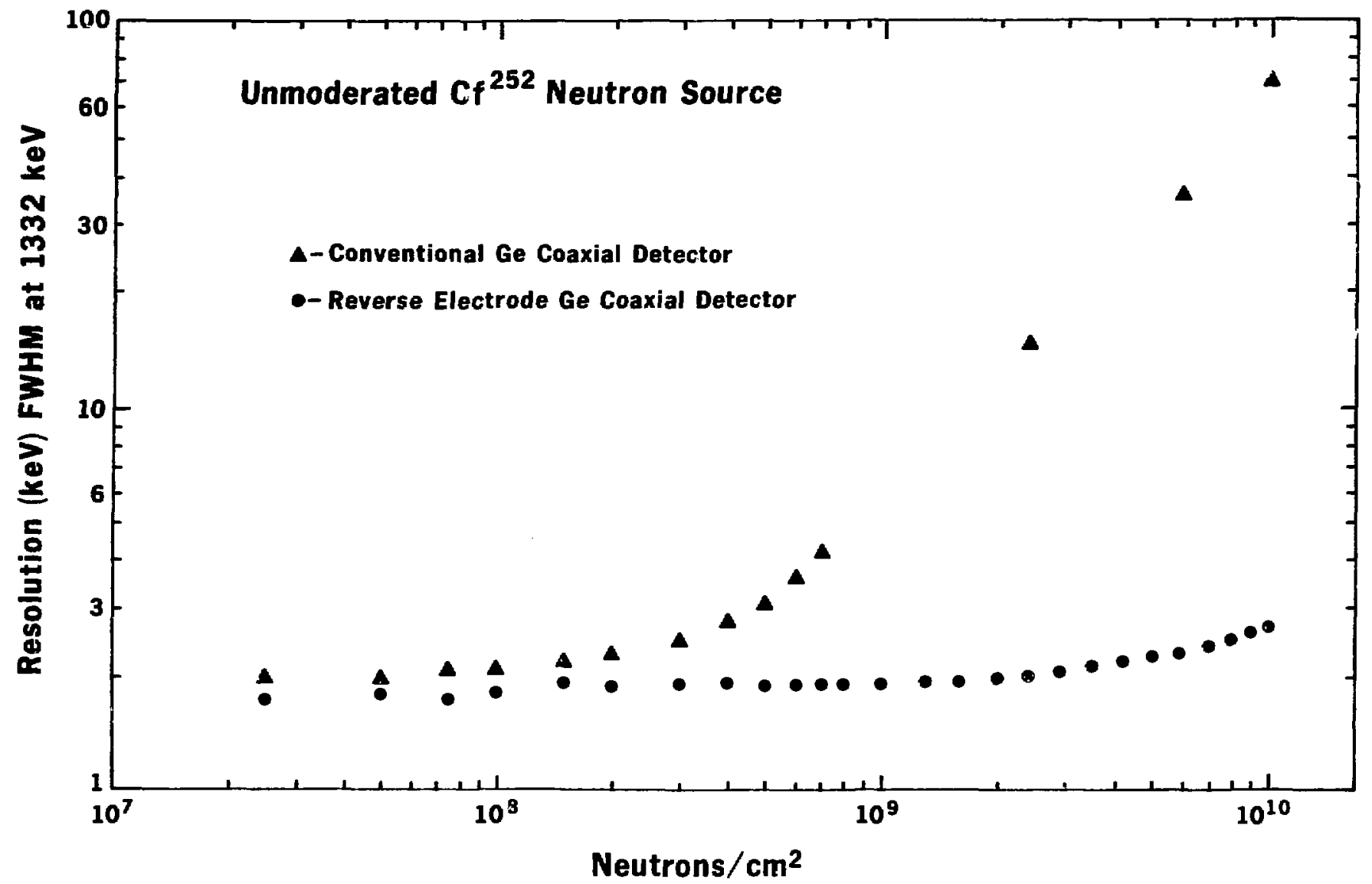

Fio. 4. Effect of neutron fluence on the eneray resolution (FI!ly') of the

$X B L \quad 7810-11754$ 1332 keV Co line for both the conventional and reverse electrode configuration Ge coaxial Hetectors. Electronic noise has not been subtracted. 


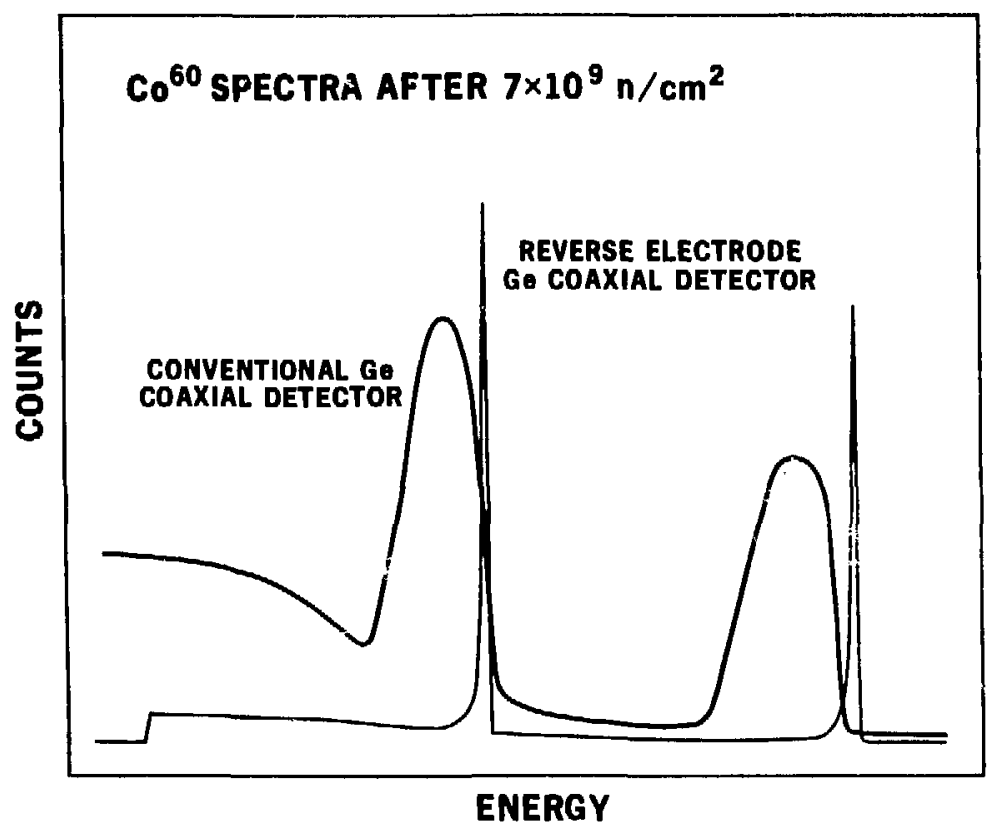

$\times$ BL $781 \mathrm{C}-11825$

Fig. 5. ${ }^{60}$ Co energy spectra obtained from both the conventional and reverse electrode configuration Ge coaxial detectors after a neutron fluence of $7 \times 10^{9} \mathrm{n} / \mathrm{cm}^{2}$. 